SNUTP 97-108

\title{
Zero Temperature Chiral Phase Transition in (2+1)-Dimensional QED with a Chern-Simons Term
}

\author{
Deog Ki Hong \\ Department of Physics, Pusan National University \\ Pusan 609-735, Korea
}

\begin{abstract}
We investigate the zero temperature chiral phase transition in $(2+1)$ dimensional QED in the presence of a Chern-Simons term, changing the number of fermion flavors. In the symmetric phase, there are no light degrees of freedom even at the critical point. Unlike the case without a Chern-Simons term, the phase transition is first-order.
\end{abstract}

11.10.Kk, 12.20.Ds, 11.15.Tk

*Email address: dkhong@hyowon.cc.pusan.ac.kr 
Recently, the phase transition at zero temperature has been studied extensively in both supersymmetric [1] and non-supersymmetric gauge theories [2 [5]. Gauge theories show a rich phase structure as one changes the number of color $N_{C}$ or the number of flavors $N_{f}$ in different representations. In a non-supersymmetric $S U\left(N_{C}\right)$ gauge theory, the phase structure is infered from the behavior of gauge coupling for different number of flavors. For large $N_{f}$, the theory is in Coulomb phase and for $N_{f}$ just below $11 N_{C} / 2$ it has an infrared fixed point [6]. As $N_{f}$ is reduced further, chiral symmetry breaking occurs. When $N_{f}$ crosses the critical point $N_{f}^{c}$ for chiral symmetry breaking, the theory exhibits discontinuity in the mass of scalar particles [3]. This discontinuity is then further investigated in view of conformal symmetry [ [i甲⿴囗十 . In this brief report, we study the zero temperature phase transition associated with dynamical mass generation in (2+1)-dimensional QED with a Chern-Simons term and will show that the mass spectrum in scalar particles does change discontinuously near the critical point, which therefore admits the conformal symmetry argument advocated

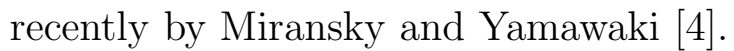

Quantum electrodynamics in three dimensions describes the high temperature limit of $(3+1)$-dimensional QED and also certain planar condensed matter systems. In studying those systems, it is important to understand how fermions get mass dynamically. Dirac mass term for two-component complex fermion in $2+1$ dimensions breaks parity $(P)$ and time reversal symmetry $(T)$, as well as the continuous flavor symmetry of the system. Pisarski found, solving the Schwinger-Dyson (SD) gap equation in $1 / N_{f}$ expansion, that dynamical mass generation occurs and the flavor symmetry $U\left(2 N_{f}\right)$ breaks down to $U\left(N_{f}\right) \times U\left(N_{f}\right)$ [7]. Appelquist et al. then analyzed the SD equation more carefully to find that there is a phase transition at a critical number of fermion flavors, below which dynamical mass generation takes place [8]. In the four-component spinor notation, in the leading order in $1 / N_{f}$ expansion, it is $32 / \pi^{2}$ in Landau gauge. Later, the exact value was found to be $128 /\left(3 \pi^{2}\right)$ [9]. Similar analysis has been done for $(2+1)$-dimensional QED with a Chern-Simons term, which allows photon to have a gauge-invariant, but $P$ and $T$ violating mass [10], and it is found that the Chern-Simons term reduces the critical number of flavor and the magnitude of dynamical fermion mass, namely the critical number of flavor becomes $\tilde{N}_{f}^{c}=N_{f}^{c} /\left[1+(\kappa / \alpha)^{2}\right]$ and the dynamical mass $m(\kappa \neq 0)=m(\kappa=0) \exp \left(-4 N_{f} / N_{f}^{c} \cdot \kappa^{2} / \alpha^{2}\right)$ [11]. It is then further investigated by Kondo and Maris [12] to show that the dynamical mass does not vanish as $N_{f}$ approaches the critical value, just like the order parameter of first-order phase transition.

As was done by Appelquist et al. [2,3], we follow the method employed by Nambu and Jona-Lasinio [13] to solve the SD equation for the fermion-antifermion scattering amplitude in the symmetric phase of (2+1)-dimensional QED with a Chern-Simons term with $N$ twocomponent complex fermions in $1 / N$ expansion. To facilitate $1 / N$ expansion, we keep $e^{2} N \equiv$ $16 \alpha$ finite when $N$ goes to infinity. We set the (Euclidean) momentum of the initial fermion and antifermion to $q / 2$, but assign momenta $q / 2 \pm p$ for the final fermion and antifermion, allowing a momentum transfer $p$ between the fermion and antifermion. We then look for a pole in (Minkowsky) $q^{2}$ for the scattering amplitude.

We take the Dirac indices of the initial fermion and antifermion as $\lambda$ and $\rho$, while the final state fermion and antifermion $\sigma$ and $\tau$. In the leading order in $1 / N$, the SD equation for the fermion-antifermion scattering amplitude is given in Euclidean space as 


$$
\begin{aligned}
& T_{\lambda \rho \sigma \tau}(p, q)=\frac{16 \alpha}{N}\left(\gamma^{\mu}\right)_{\sigma \lambda} D_{\mu \nu}(p)\left(\gamma^{\nu}\right)_{\rho \tau} \\
& \quad+\frac{16 \alpha}{N} \int_{k} T_{\lambda \rho \sigma^{\prime} \tau^{\prime}}(k, q)\left(\gamma^{\mu} \frac{1}{\frac{1}{2} q^{\prime}+\not h}\right)_{\sigma \sigma^{\prime}} D_{\mu \nu}(p-k)\left(\frac{1}{-\frac{1}{2} q^{\prime}+\not \phi^{\prime}} \gamma^{\nu}\right)_{\tau^{\prime} \tau},
\end{aligned}
$$

where $D_{\mu \nu}(p)$ is the photon propagator and Dirac gamma matrices $\gamma^{\mu}$ in three dimensions are just the Pauli matrices satisfying $\operatorname{tr}\left(\gamma^{\mu} \gamma^{\nu} \gamma^{\lambda}\right)=2 i \epsilon^{\mu \nu \lambda}$. The photon propagator in $1 / N$ expansion is given by summing up all the bubble diagrams as

$$
D_{\mu \nu}=\left[g_{\mu \nu}-p_{\mu} p_{\nu} / p^{2}\right] \Pi_{1}(p)+\epsilon_{\mu \nu \lambda} p^{\lambda} \Pi_{2}(p)
$$

where we choose Landau gauge and

$$
\begin{aligned}
& \Pi_{1}(p)=\frac{|p|+\alpha}{|p|\left[(|p|+\alpha)^{2}+\kappa^{2}\right]} \\
& \Pi_{2}(p)=\frac{1}{p^{2}} \cdot \frac{\kappa}{(|p|+\alpha)^{2}+\kappa^{2}}
\end{aligned}
$$

with $\kappa$ the coefficient of the Chern-Simons term. (Note that in general there will be a one-loop correction to the Chern-Simons term but we have chosen a regulator such that the correction will be of the order of $1 / N$.) Since the direct product of two spinors in $2+1$ dimensions is either scalar or vector, we may write the scattering amplitude as

$$
T_{\lambda \rho \sigma \tau}=\delta_{\lambda \rho} \delta_{\sigma \tau} T+\delta_{\lambda \rho}\left(\gamma^{\alpha}\right)_{\sigma \tau} T_{\alpha}+\left(\gamma^{\alpha}\right)_{\lambda \rho} \delta_{\sigma \tau} T_{\alpha}^{\prime}+\left(\gamma^{\alpha}\right)_{\lambda \rho}\left(\gamma^{\beta}\right)_{\sigma \tau} T_{\alpha \beta}
$$

where $T$ is the scalar channel amplitude, $T_{\alpha}$ vector channel, and so on. Further, we write the vector channel amplitude as

$$
T^{\alpha}(p, q)=i p^{\alpha} T_{1}(p, q)+i q^{\alpha} T_{1}^{\prime}(p, q) .
$$

For small $q$ limit, the second term in Eq. (5) is negligible .

Plugging Eq. (雨) into the SD equation, one finds the scalar channel amplitude $T$ is coupled to the vector channel amplitude $T^{\alpha}$. We consider $p \gg q$. Then $q$ will be simply act as an infrared cutoff in the loop integrals. Multyplying $\delta_{\lambda \rho} \delta_{\sigma \tau} / 4$ and $\delta_{\lambda \rho} \gamma_{\tau \sigma}^{\alpha} / 4$ respectively to Eq. (1), and integrating the angular variables, we get for $p \ll \alpha$

$$
\begin{aligned}
T(p, q)= & \frac{16}{N_{0} p}+\frac{8}{N_{0} \pi^{2}} \int_{q}^{\infty} \frac{d k}{p k} T(k, q)(p+k-|p-k|) \\
& -\frac{4}{N_{1} \pi^{2}} \int_{q}^{\infty} d k\left[\frac{p^{2}-k^{2}}{2 p k} \ln \left(\frac{p+k}{|p-k|}\right)\right] T_{1}(k, q) \\
T_{1}(p, q)= & -\frac{16}{N_{1} p^{2}}-\frac{8}{N_{1} \pi^{2} p^{2}} \int_{q}^{\infty} d k\left[\frac{p^{2}-k^{2}}{2 p k} \ln \left(\frac{p+k}{|p-k|}\right)\right] T(k, q)
\end{aligned}
$$

where $p$ and $q$ denote the magnitude of momentum and

$$
N_{0}=N \cdot \frac{\alpha^{2}+\kappa^{2}}{\alpha^{2}}=N_{1} \cdot \frac{\kappa}{\alpha} .
$$


When the Chern-Simons term is absent, $(2+1)$-dimensional QED is finite in the ultraviolet (UV) region and the integral in Eq. (11) will be rapidly damped for $k>\alpha$. With a Chern-Simons term, the UV structure of $(2+1)$-dimensional QED may be quite different. Especially, in the broken phase, the Chern-Simons term will dominate in the ultraviolet region, since the fermion mass function falls off slowly [12]. But, in the symmetric phase, where we are interested in, the parity-even mass function is zero and in the photon propagator, Eq. (2), the parity-odd part falls off more rapidly than the parity-even part. Therefore, the integral in Eq. (1) falls off rapidly for $k>\alpha, \kappa$. Therefore, we can take $\alpha$ as the UV cutoff and assume $\kappa \simeq \alpha$. As approximation, we further take

$$
\frac{p^{2}-k^{2}}{2 p k} \ln \left(\frac{p+k}{|p-k|}\right) \simeq \theta(p-k)-\theta(k-p)
$$

which should give a good approximation since the error in the integration will be the order of $p / \alpha$.

Now, we can convert the coupled integral equations, Eq.'s (6) and (8), into coupled differential equations:

$$
\begin{aligned}
\frac{d^{2}}{d p^{2}}(p T) & =-\frac{16}{N_{0} \pi^{2} p} T-\frac{8}{N_{1} \pi^{2} p} \frac{d}{d p}\left(p^{2} T_{1}\right) \\
\frac{d}{d p}\left(p^{2} T_{1}\right) & =-\frac{16}{N_{1} \pi^{2}} T .
\end{aligned}
$$

We see that the vector channel amplitude is subdominant by $1 / N_{1}$, compared to the scalar channel amplitude, and so is the effect of the Chern-Simons term to the scalar amplitude. In $1 / N$ expansion, we can neglect the second term in the right-hand side of Eq. (11) and the scalar channel amplitude is decoupled;

$$
\frac{d^{2}}{d p^{2}}(p T)=-\frac{16}{N_{0} \pi^{2} p} T
$$

which admits a power solution,

$$
T(p, q)=\frac{A(q)}{\alpha}\left(\frac{p}{\alpha}\right)^{-(1 / 2)+(1 / 2) \eta}+\frac{B(q)}{\alpha}\left(\frac{p}{\alpha}\right)^{-(1 / 2)-(1 / 2) \eta},
$$

where $\eta=\sqrt{1-\tilde{N}_{f}^{c} / N}$ and $\tilde{N}_{f}^{c}=\frac{64}{\pi^{2}} /\left[1+(\kappa / \alpha)^{2}\right]$. (Note in the four-component spinor notation $\tilde{N}_{f}^{c}$ has to be reduced by $1 / 2$.) With the solution for $T$, we find from Eq. (12)

$$
T_{1}(p, q)=\frac{C_{1}(q)}{\alpha^{2}}\left(\frac{p}{\alpha}\right)^{-2}+\frac{A_{1}(q)}{\alpha^{2}}\left(\frac{p}{\alpha}\right)^{-(3 / 2)+(1 / 2) \eta}+\frac{B_{1}(q)}{\alpha^{2}}\left(\frac{p}{\alpha}\right)^{-(3 / 2)-(1 / 2) \eta}
$$

where

$$
\begin{aligned}
& A_{1}(q)=-\frac{32}{N_{1} \pi^{2}} \frac{A(q)}{1+\eta}, \quad B_{1}(q)=-\frac{32}{N_{1} \pi^{2}} \frac{B(q)}{1-\eta} \\
& C_{1}(q)=-\frac{16}{N_{1}}+\frac{16}{N_{1} \pi^{2}}\left\{\frac{A(q)}{1+\eta}\left[\left(\frac{q}{\alpha}\right)^{(1 / 2)+(1 / 2) \eta}+1\right]+\frac{B(q)}{1-\eta}\left[\left(\frac{q}{\alpha}\right)^{(1 / 2)-(1 / 2) \eta}+1\right]\right\} .
\end{aligned}
$$


The $q$ dependence of the amplitudes can be determined by substituting the solutions back into Eq. (6). This gives

$$
\begin{aligned}
& A(q)=\frac{(1+\eta) \pi^{2} / 2(q / \alpha)^{-(1 / 2)+(1 / 2) \eta}}{(q / \alpha)^{\eta}-[(1+\eta) /(1-\eta)]^{2}} \\
& B(q)=-\frac{1+\eta}{1-\eta} A(q) .
\end{aligned}
$$

We see that the pole structure of the scalar and vector channel amplitudes in $(2+1)$ dimensional QED is same whether or not the Chern-Simons term is present, except that the exponent $\eta$ changes due to the change in the critical number of fermion flavors. The location of the poles of the amplitudes in the complex $q$ plane is at $q=q_{0}$ with

$$
\left|q_{0}\right|=\alpha\left(\frac{1+\eta}{1-\eta}\right)^{2 / \eta} .
$$

When $N$ approaches the critical number $\left(N \rightarrow \tilde{N}_{f}^{c}\right), \eta \rightarrow 0$ and $\left|q_{0}\right| \rightarrow \alpha \exp (4)$, which is beyond the domain of our approximation, $q \ll \alpha$. The mass of the scalar and vector bound states does not vanish as $N$ approaches the critical value, while in the broken phase the scalar bound state mass is zero due to Goldstone theorem. We find therefore that, with or without a Chern-Simons term, $(2+1)$-dimensional QED exhibits discontinuity in the scalar mass spectrum. This finding shows that there is no long range correlation in symmetric phase, because both photons and fermions also get parity-odd mass due to the ChernSimons term. Since the order parameter in the broken phase for the zero temperature chiral phase transition does not vanish even at the critical point, we see that the phase transition associated with dynamical mass generation behaves as a regular first-order phase transition. This is in sharp contrast to pure QED3, which exhibits a peculiar phase transition; namely, in pure QED3, the order parameter in the broken phase vanishes at the critical point while there is no scalar whose physical mass approaches zero at the critical point in the symmetric phase. In Ref. [2], Appelquist et al. argued that the peculiarity of the phase transition can be attributed to the fact that the effective potential is not analytic at zero dynamical mass, $m$, of the fermion at zero momentum because the theory has a long range force mediated by gauge fields. Here, we note that the regular first-order phase transition in QED3 with a Chern-Simons term supports this argument because the effective potential is analytic at $m=0$ when a Chern-Simons term is present; there is only a finite number of oscillating solutions to the SD gap equation with the Chern-Simons term [12,14] and there is no long range force.

To conclude, we have studied the zero temperature chiral phase transition in QED3 with a Chern-Simons term by solving a Schwinger-Dyson equation for the fermion-antifermion scattering amplitude in the symmetric phase $\left(N>\tilde{N}_{f}^{c}\right)$ in $1 / N$ expansion. We find that as in pure QED3 no light degrees of freedom appear in the symmetric phase, which implies that the phase transition is first-order because the order-parameter does not vanish in the broken phase even at the critical point. Since the effective potential is analytic in the dynamical fermion mass at zero momentum, this result supports the argument of Appelquist et al. that the peculiar phase transiton at zero temperature in pure QED3 is due to the non-analyticity of the effective potential at zero dynamical fermion mass. 


\section{ACKNOWLEDGMENTS}

We thank T. Appelquist for useful discussions. This work was supported in part by the KOSEF through SRC program of SNU-CTP and also by Basic Science Research Program, Ministry of Education, 1996 (BSRI-96-2413). 


\section{REFERENCES}

[1] For recent review, see K. Intriligator and N. Seiberg, hep-th/9509066, Nucl. Phys. B(PS) 45B,C, 1 (1996).

[2] T. Appelquist, J. Terning, and L.C.R. Wijewardhana, Phys. Rev. Lett. 75, 2081 (1995).

[3] T. Appelquist, J. Terning, and L.C.R. Wijewardhana, Phys. Rev. Lett. 77, 1214 (1996).

[4] V. Miransky and K. Yamawaki, Phys. Rev. D 55, 5051 (1997).

[5] R.S. Chivukula, Phys. Rev. D 55, 5238 (1997).

[6] T. Banks and A. Zaks, Nucl. Phys. B196, 189 (1982).

[7] R. Pisarski, Phys. Rev. D 29, 2423 (1984).

[8] T. Appelquist, D. Nash, and L.C.R. Wijewardhana, Phys. Rev. Lett. 60, 2575 (1988).

[9] D. Nash, Phys. Rev. Lett. 62, 3024 (1989).

[10] S. Deser, R. Jakiw, and S. Templeton, Ann. Phys. (N.Y.) 140, 372 (1982).

[11] D.K. Hong and S.H. Park, Phys. Rev. D 47, 3651 (1993).

[12] K.-I. Kondo and P. Maris, Phys. Rev. Lett. 74 18, (1995); Phys. Rev. D 52, 1212 (1995).

[13] Y. Nambu and G. Jona-Lasinio, Phys. Rev. 122, 345 (1961).

[14] T. Ebihara et al. Nucl. Phys. B 434, 85, (1995). 